\title{
CoViD-19 Pandemic and Bariatric Surgery in Argentina
}

\author{
Axel F. Beskow ${ }^{1,2}$ (D) $\cdot$ Pedro R. Martinez-Duartez ${ }^{2,3} \cdot$ Estuardo J. Behrens Estrada $^{4} \cdot$ Felipe E. Fiolo $^{2,5} \cdot$ Almino C. Ramos $^{6}$
}

Received: 15 May 2020 /Revised: 20 September 2020 / Accepted: 23 September 2020 / Published online: 6 October 2020

(C) Springer Science+Business Media, LLC, part of Springer Nature 2020

\begin{abstract}
Argentina was able to anticipate public health interventions in order to flatten the contagion curve of CoViD-19. Eighty-three surgeons answered an online survey to assess the impact of the pandemic on bariatric surgery (BS) in Argentina. Most of them showed a high economic dependence on BS. Near $90 \%$ of health institutions were on phase 0 or I. While $90 \%$ still performed other laparoscopic surgeries, BS was suspended. In many surgeries for nonsuspected CoViD-19 patients, high personnel protection resources were applied. Ninety-five percent offered virtual consults. Most surgeons would not change usual algorithms or techniques. To restart BS a scientific society recommendation was expected, including patient selection criteria. The opinions gathered by this survey were taken into account to elaborate official recommendations for restarting elective BS.
\end{abstract}

Keywords Bariatric surgery $\cdot \mathrm{CoViD}-19$ pandemic $\cdot$ SARS-CoV2 $\cdot$ Public health regulations $\cdot$ Personal protective equipment

\section{Introduction}

The SARS-CoV-2 virus pandemic has specific characteristics that have led to its rapid global dispersion, jeopardizing all levels of health services in the affected countries [1-3]. Obesity [4-8] and, in particular, BMI over 35, is a risk factor for a severe CoViD-19 infection, even more significant than other conditions such as diabetes or hypertension. Clearly, measures such as social distancing and reinforcement of personal hygiene habits reduce the possibility of transmission.

The state of the pandemic in Latin America differs from that of the northern hemisphere [9]. Time and distance allowed for the implementation of public health interventions at an early phase with the aim of flattening the curve and preparing the health system to properly address the peak intensity of the epidemic. The first case in Argentina was reported on March 3rd, 2020, and by March 19 the national government had issued mandated social distancing by decree, which suspended virtually all economic activities. By that date, 30 new cases had been detected, mostly from people that had traveled abroad. These regulations resulted in a stagnation in the number of new infections with a bigger-than-expected flattening of the curve, and a virtual halt on all programmed healthcare services, including face-to-face consults and most elective surgeries. This situation became widespread throughout the vast territory of the country.
Axel F. Beskow

axel.beskow@hospitalitaliano.org.ar

Pedro R. Martinez-Duartez

pedromartinez23@hotmail.com

Estuardo J. Behrens Estrada

ebehrens@comnetsa.com

Felipe E. Fiolo

fefiolo@gmail.com

Almino C. Ramos

ramos.almino@gmail.com
1 Bariatric Surgery at Hospital Italiano de Buenos Aires, Ciudad Autónoma de Buenos Aires, Argentina

2 Argentine Society for the Surgery of Obesity (SACO), Bolivar 3585, Mar del Plata B7600GEA, Argentina

3 Bariatric Surgery at Hospital Universitario Austral, Buenos Aires, Argentina

4 Medical Director of New Life Center, Bariatric Surgery Institute, Guatemala City, Guatemala

5 Bariatric Surgery at Hospital Privado de Comunidad, Mar del Plata, Buenos Aires, Argentina

6 Gastro-Obeso-Center Institute of Metabolic Optimization, Sao Paulo, Brazil 
One month after these restrictive measures, CoViD-19 had accumulated 3031 cases (19\% regarded as community transmissions) and 142 fatalities. A few days before, the Latin American Chapter of IFSO (IFSO LAC) and the local Argentine Society for the Surgery of Obesity (SACO, for its acronym in Spanish) conducted a survey among bariatric surgeons to determine the impact of the pandemic and the measures undertaken on the practice of bariatric surgery in Argentina.

The objective of this study is to report on the findings of the survey as well as to outline safe practices required to resume elective bariatric surgery.

\section{Methods}

On April 17, 2020, a survey was distributed among bariatric surgeons residing in Argentina. The survey, entitled "CoViD19 and Bariatric Surgery in Latin America", was created online using Google Forms and is available as an Annex to this document (Annex 1). The survey was anonymous. All surveys were received between April 17 and 21.

\section{Results}

Eighty-three surgeons answered the survey. Thirty-one percent of the respondents inhabited the Autonomous City of Buenos Aires, while an additional third were from its surrounding provinces, Buenos Aires, Santa Fe, and Córdoba. No answers were received from five provinces: Chaco, Jujuy, La Rioja, Catamarca, or Chubut.

During 2019, the 83 surgeons performed a total of 10,515 bariatric surgeries, averaging 126.7 surgeries per surgeon. More than $65 \%$ of surgeons stated that more than $50 \%$ of their income derives from their bariatric practice, and $41 \%$ stated that more than $75 \%$ of their income does.

Hospitalization for patients varied between 12 and $72 \mathrm{~h}$, with an average of $38.4 \mathrm{~h}$ (1.6 days). Eighty-five percent of the respondents informed ICU usage for less than $1 \%$ of their patients, while $7 \%$ for less than $5 \%$ of their cases.

According to the SAGES descriptions on the institutional CoViD-19 phases (https://www.sages.org/sagesrecommendations-surgical-management-gastric-cancercovid-19-crisis/), $54.2 \%$ reported being on phase I, and $33.7 \%$ had yet to be affected by the pandemic. Only $4.8 \%$ reported being on phase II. All surgeons, except for one, suspended all elective bariatric surgery, with a mean of 7.7 days prior to the first regional case. Ninety percent of the respondents continue performing other types of laparoscopic surgeries on patients without suspected SARS-CoV-2 virus infection.

Seventy-five surgeons (90\%) reported access to endoscopy being limited, mostly for medical emergencies only.
However, only $26.5 \%$ would consider performing bariatric surgery without a previous endoscopic evaluation.

Seventy-nine $(95.2 \%)$ reported offering their patients virtual follow-up consults, although $48 \%$ do not expect to obtain retribution of any kind for these.

All surgeons referred performing some sort of screening for the detection of SARS-CoV-2 virus infection in asymptomatic patients, regardless of their medical histories. Most opted for chest imaging of different types (90.4\%), while only $55.4 \%$ requested PCR testing.

The survey inquired about several personal protection measures and other measures related to pneumoperitoneum to be taken in the event of performing surgery. It was noticeable that $79.5 \%$ of surgeons would use face masks with high filtration efficiency (N95-rated or more), and 56.6\% face shields, while two-thirds would use gas filters for the pneumoperitoneum, on patients without suspected SARS-CoV-2 infection.

Two-thirds of the surgeons will only return to their activities when the pandemic is resolved, while almost $90 \%$ predict that their activities will be greatly reduced, and most even anticipate that by as much as $50 \%$ (Fig. 1).

Exploring the conditions deemed necessary to restart elective procedures, three were proposed: institutions in phase 0 or I, and personal protection requirements fulfilled; patient selection; and scientific society approvement of elective surgery. Forty-seven surgeons $(56.6 \%)$ agreed that the three conditions should be met, while $20.5 \%$ regarded the scientific society's recommendation as enough. The criteria for selecting patients which achieved a greater consensus were absence of those known risk factors for viral contagion, the absence of chronic lung disease (only history of asthma was taken into account), age under 60, and immunological integrity (assessed as the need for immunosuppressant drugs). Other conditions, which refer to the severity of comorbidities, did not reach consensus as criteria for selection (Fig. 2).

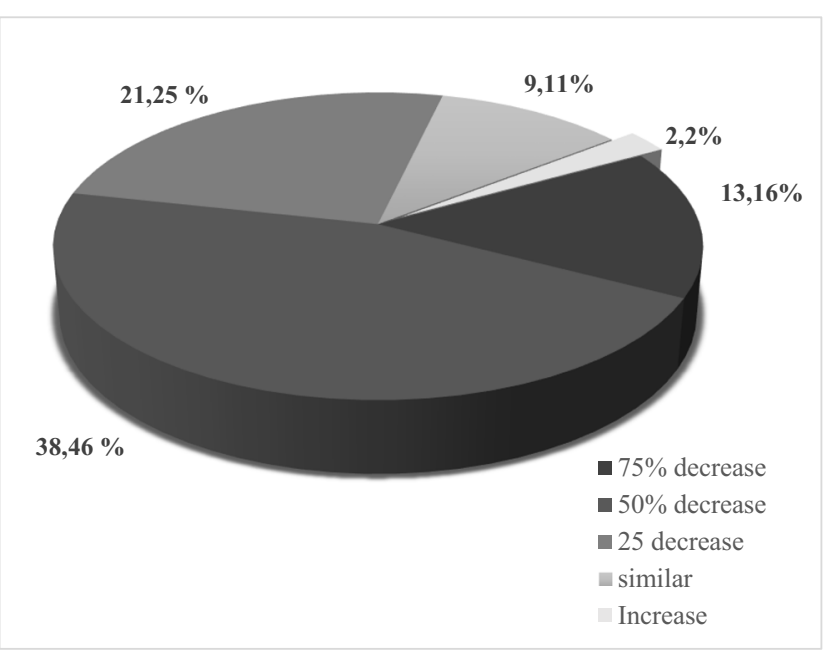

Fig. 1 Estimated future variation in bariatric activity in the medium term 
Fig. 2 Patient selection criteria for elective bariatric surgery in the context of COVID-19 pandemic (OSA: obstructive sleep apnea)

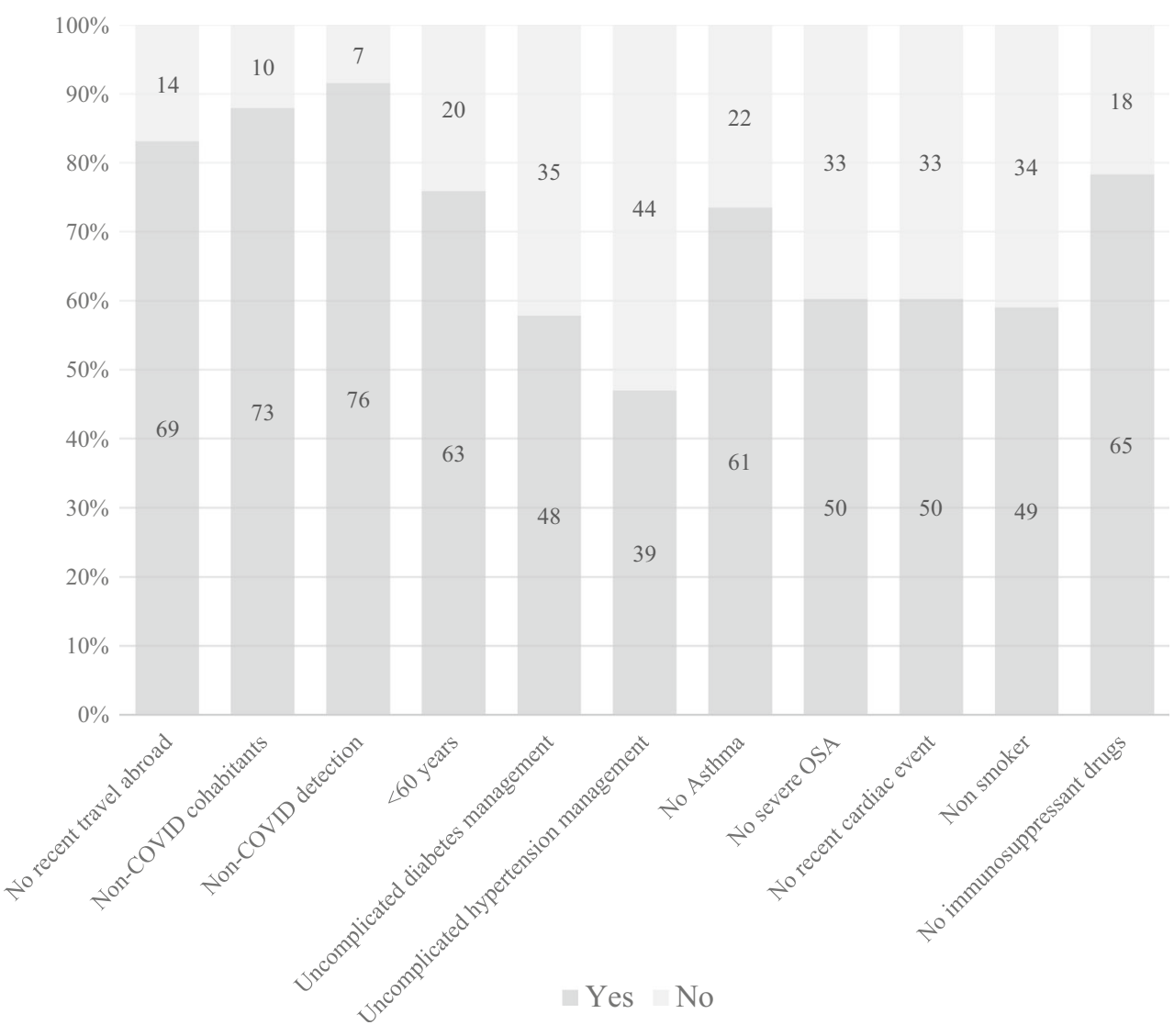

Only $19.2 \%$ of surgeons agreed that a sleeve gastrectomy could be an ideal procedure within the pandemic, while $87.9 \%$ would not be willing to change their chosen technique based on an infection risk.

The opinions gathered by this survey were taken into account to elaborate the SACO recommendations for an eventual return of elective surgery activity, published on May 7th, and available at http://sacobariatrica.org/recomendacioncovid/.

\section{Discussion}

It is clear that CoViD-19 has altered normal life almost all over the planet. Overall, the extent of this disturbance is due to individual fear and the measures undertaken at government level, which are in proportion to the local impact of the pandemic. However, it may happen that in early stages, where the immediate evolution will take the problem is unknown, restrictive measures are put in place to ensure the health of the community. In Argentina, this has been the situation, which has paralyzed the programmed medical care. The flattening result of the contagion and death curve should not be devaluated, since it allowed preparing the country for a worse scenario. Bariatric surgery, like almost all scheduled surgery, was suspended in this context. A month after the mandatory quarantine began, the SACO was urged to prepare recommendations to restart the activity.

Through the survey analyzed in this work, important information was obtained on the impact of the pandemic on bariatric activity at the national level and on the opinions of a good number of bariatric surgeons, most of whom are highly dependent on their economy for this activity. The pessimistic view of the majority on the impact they will suffer and on the possibilities of restarting this type of surgery did not prevent them from seeking the resources to maintain adequate follow-up of their patients, nor did it make them change their usual preoperative algorithms.

It is striking how argentine surgeons were influenced by the experiences collected in Europe and the USA, where the impact of the pandemic was much more serious, raising the level of surgical personnel protection. This became evident in the decision of the majority to use costly n95 masks, or more than $50 \%$ to use pneumoperitoneum gas filters, even in surgeries of patients not suspected of CoViD-19. Although it could be explained by the lack of availability of specific tests, it seems disproportionate to the level of community circulation of the virus in Argentina.

But the most relevant data from the survey is the consensus that certain conditions are required for restarting elective bariatric surgeries, and of these, the recommendation by the bariatric society was the most expected. The institutional 
situation came in second place. Third, patient selection focused on avoiding operating asymptomatic CoViD-19positive patients, followed by excluding individuals at higher risk for viral disease due to age, chronic pulmonary disease, or immunosuppression.

With these opinions, and supported on international recommendations and the consensus of experts, the local society elaborated the current recommendations for elective bariatric surgery in Argentina, which are acknowledged throughout the country, with different implementation depending on the regional epidemic situation. The recommendations do not detail the type of procedure selection criteria, nor a modality for prioritizing patients, as do most of the opinions collected in the survey.

\section{Conclusions}

The CoVid-19 pandemic severely affected bariatric surgery activity in Argentina, generating a pessimistic vision of its possible normalization, and forced an adaptation of the surgeons' practice. An online survey was a rapidly applicable and useful resource for obtaining specific information on the situation of bariatric surgery, and for preparing official safe restart recommendations, according to local reality and with a high level of consensus in Argentina.

\section{Compliance with Ethical Standards}

All procedures performed in the study involving human participants were in accordance with the 1964 Helsinki declaration and its later amendments or comparable ethical standards.

Informed consent was obtained within the survey from all individual voluntary participants included in the study.

Conflict of Interest The authors declare that they have no conflict of interest.

\section{Annex}

Important: This is the translation of a survey originally conducted in Spanish.

COVID 19 and Bariatric surgery in LATAM

We send you a cordial greeting from IFSO LAC, hoping that you and your valued families are well.

In order to know well the current situation of the COVID-19 pandemic in Latin America, we ask that you please take a few minutes to fill out this survey. It is designed to know the current status of each member country of IFSO LAC, and thus we can propose, according to the results we obtain, some management guides, and be able to project ourselves to know when and how we should start scheduling elective patients. We know very well that this pandemic has affected each region differently, so it is necessary that we manifest ourselves as a chapter.
1. I agree to voluntarily participate in the study, allowing the data obtained to be used in any publication and elaboration of society recommendations for restarting elective bariatric surgery. (Yes/ No).

2. Age (number).

3. City where you work (name).

4. What percentage of your income does bariatric surgery represent for you? (one option)

- $\quad$ Less than $25 \%$

- Between 26 to $49 \%$

- Between 50 to $74 \%$

- Between 75 to $94 \%$

- More than $95 \%$

5. How many bariatric surgeries did you do in 2019? (number).

6. What percentage of your bariatric surgery patients do you send to the ICU? (one option)

- Never

- Less than $1 \%$

- Less than $5 \%$

- Less than $10 \%$

- All patients per protocol go to ICU

7. What is the average hospital stay of your bariatric surgery patients? (one option)

- $\quad 12$ to 14 hours

- 1 day

- 2 days

- 3 days

-4 days

8. In what type of institution do you perform most bariatric procedures? (one option)

- Public Hospital

- Private Hospital

- Both

- University Public Hospital or teaching hospital.

- Private University Hospital or teaching hospital.

9. Number of beds in your institution? (one option)

$-\quad<100$

- 100-200

- 201-500

- 501-1000

$-\quad>1000$ 
10. Based on the SAGES COVID-19 phases classification, what phase is your hospital in? (one option)

- Phase 0

- Phase 1

- Phase 2

- Phase 3

11. Did you have COVID-19 cases in your region where you operate bariatric? (Yes/No)

12. When did the first COVID-19 case occur in the region where you perform bariatric surgery? (date).

13. Did the government authorities declare a quarantine in your city, leaving only essential services? (Yes/ No).

14. Have your government authorities decreed that elective surgeries CANNOT be performed in your city? (Yes/No).

15. From what date and until when is the quarantine? (date 1-date 2).

16. Did your local scientific society recommend that you stop elective bariatric surgery? (Yes/No).

17. Do you still perform elective bariatric surgery today? (Yes/No).

18. When was your last elective bariatric surgery? (date).

19. How long do you think you will need to postpone elective surgeries due to the COVID-19 pandemic? (one option)

- I did not postpone elective surgeries.

- 1 month

- 2 month

- 3 month

- 4 month

- An estimated time cannot be given yet.

20. How do you think the pandemic will affect bariatric surgery (BS)? (one option)

- The volume of the BS will increase

- It will not impact the volume of the surgery.

- It will decrease up to $25 \%$ of the BS

- It will decrease up to $50 \%$ of the BS

_ It will decrease up to $75 \%$ of the BS

We refer to any type of surgery not only bariatric.

21. If, before COVID-19, you did a surgical technique laparoscopically, today which approach would you use for the same technique? (one option)

- We continue using the laparoscopic approach.

- We changed from laparoscopy to conventional surgery.
22. If you have to operate on an asymptomatic patient with any pathology, do you routinely perform a screening to rule out SARS-CoV-2? (Yes/No).

23. What COVID-19 screening studies do you perform in addition to history and symptoms evaluation? (may be more than one option)

- Chest X-ray

- Chest CT scan

- Serology

- PCR

- Quick test.

- Ultrasound

24. If you have to do surgery today, in an asymptomatic patient (Not suspicious or COVID positive), check the personal protection measures that the surgeon, assistants and instrumenter would use: (may be more than one option)

- All the same as before the COVID-19 pandemic

- N95, FFP2 or FFP3 mask

- Goggles

- Face shield

- Gas filters for the insufflated pneumoperitoneum

- Gas filters for exsufflated pneumoperitoneum

- Commercial smoke evacuation systems

- Negative pressure operating room

25. If you have to do surgery on a COVID positive patient. Check the personal protection measures that the surgeon, assistants and instrumenter would use: (may be more than one option)

- $\quad$ All the same as before the COVID-19 pandemic

- N95, FFP2 or FFP3 mask

- Goggles

- Face shield

- Gas filters for the insufflated pneumoperitoneum

- Gas filters for exsufflated pneumoperitoneum

- Commercial smoke evacuation systems

- Negative pressure operating room

26. Did you operate on COVID patients? (Yes/No).

27. During this time of the pandemic, in which cases do you perform endoscopies at your institution? (one option)

- Only emergency

- Elective and emergency

- Endoscopies are not done due to the pandemic

28. If you routinely perform preoperative endoscopy to evaluate your patient candidate for bariatric surgery, at this 
time of the COVID-19 pandemic, what will you do? (one option)

- Continue doing endoscopy pre-operatively

- Change my protocol and request only a barium swallow study

- Change my protocol, I will NOT request endoscopy nor barium swallow study

29. Do you continue to do face-to-face consultations? (Yes/ No).

30. Since when have you stopped face-to-face consultations with your patients? (date).

31. In orden to continue with pre- and post-operative treatment, does your multidisciplinary team offer online or telemedicine services? (Yes/No).

32. In what percentage can you charge the fees for online consultations to health insurance? (one option)

$-\quad 0 \%$

$-\quad 25 \%$

$-50 \%$

$-75 \%$

$-\quad 100 \%$

Bariatric Surgery in the context of the COVID-19 Pandemic

33. Will you change your preferred bariatric surgery technique for another in this time of COVID? (For example: change Roux-en-Y gastric bypass for sleeve gastrectomy) (one option)

- Yes

- No

- Do not know

34. Do you agree that sleeve gastrectomy could be the ideal surgery at this time of the pandemic? (Yes/No).

35. Does any of your bariatric surgery patients got COVID19? (Yes/No).

36. How many of your patients undergoing bariatric surgery got COVID-19? (number).

37. Before restarting elective bariatric surgery, what would you expect? (one option).

A) That your hospital is in phase 0 or I and the personal protection resources are available.

B) That the patient meets certain criteria to define (for example that proposed by Angrisiani or by MeNTS Score).

C) That the scientific society of your region recommend it.
D) All of the above (A, B and C).

E) A and B.

F) I would wait for the pandemic to end or for a specific vaccine or drug therapy to exist.

38. What conditions should a patient meet for elective bariatric surgery at this time? (may be more than one option)

- Be asymptomatic (no flu symptoms, no fever, no cough, shortness of breath, no gastrointestinal symptoms)

- Not having travelling in the last 15 days to countries with high viral circulation

- No recent olfactory or taste disturbances

- No history of positive COVID-19.

- Age less than 60 years

- Without diabetes that is difficult to manage or requires a lot of medication to be controlled.

- Without arterial hypertension that is difficult to manage or that requires a lot of medication to be controlled.

- Without asthma.

- Without severe obstructive sleep apnea.

- No recent heart disease.

- Non smoker.

- Performing social isolation for 15 days after surgery.

- Wearing a surgical or homemade mask at home just like the people around you.

- Without drugs that affect the patient immunologically.

- Do not live with COVID positive cases

\section{References}

1. Guan WY, Ni ZY, Hu Y, et al. Clinical characteristics of coronavirus disease 2019 in China. N Engl J Med. 2020;382:1708-20.

2. Huang C, Wang Y, Li X, et al. Clinical features of patients infected with 2019 novel coronavirus in Wuhan, China. Lancet. 2020;395: 497-506.

3. Wu Z, McGoogan JM. Characteristics of and important lessons from the coronavirus disease 2019 (COVID-19) outbreak in China: summary of a report of 72,314 cases from the Chinese Center for Disease Control and Prevention. JAMA. 2020;323:1239-42.

4. Simonnet A, Chetboun M, Poissy J, et al. High prevalence of obesity in severe acute respiratory syndrome coronavirus-2 (SARS-CoV-2) requiring invasive mechanical ventilation [published online ahead of print, 2020 Apr 9]. Obesity (Silver Spring). 2020; https://doi.org/10. 1002/oby.22831.

5. Lighter J, Phillips M, Hochman S, et al. Obesity in patients younger than 60 years is a risk factor for Covid-19 hospital admission [published online ahead of print, 2020 Apr 9]. Clin Infect Dis. 2020: ciaa415. https://doi.org/10.1093/cid/ciaa415.

6. Richardson S, Hirsch JS, Narasimhan M, et al. Presenting characteristics, comorbidities, and outcomes among 5700 patients hospitalized with COVID-19 in the New York City area. JAMA Published 
online April 22, 2020:2052. https://doi.org/10.1001/jama.2020. 6775.

7. Kass DA, Duggal P, Cingolani O. Obesity could shift severe COVID-19 disease to younger ages. Published:May 04, 2020. Lancet. https://doi.org/10.1016/S0140-6736(20)31024-2.

8. Groups at Higher Risk for Severe Illness. https://www.cdc.gov/ coronavirus/2019-ncov/need-extra-precautions/groups-at-higherrisk.html. Accessed 13 May 2020.
9. John Hopkins University and Medicine Coronavirus Resource Center. Coronavirus COVID-19 global cases. Available at: https:// coronavirus.jhu.edu/map.html. Accessed 13 May 2020.

Publisher's Note Springer Nature remains neutral with regard to jurisdictional claims in published maps and institutional affiliations. 